\title{
Modeling the Resonant Behavior of Continuously Reconfigurable FSS Based on Four Arms Star Geometry
}

\author{
Alfredo Gomes Neto (D), Jefferson Costa e Silva (D), Amanda Gomes Barboza (D), Ianes Barbosa \\ Grécia Coutinho $\mathbb{D}$, Marina de Oliveira Alencar $\mathbb{D}$, Mylenna Correia de Andrade \\ Group of Telecommunications and Applied Electromagnetism, GTEMA \\ Federal Institute of Paraiba, IFPB \\ alfredogomes@ifpb.edu.br,jefferson@ifpb.edu.br,amanda_bgomees@hotmail.com,ianesgrecia@gmail.com, \\ marina.alencar.93@gmail.com,mylennaca@gmail.com
}

\begin{abstract}
A modeling of the resonant behavior of a continuously reconfigurable frequency selective surface, FSS, is described in this paper. The FSS is based on four arms star geometry and its reconfigurability is achieved by the use of varactors. FSS, four arms star geometry and varactor principles are explained. Considering available a set of measured or numerical data, with frequency responses for different varactor capacitance values, the FSS equivalent circuit is stablished. Then, the varactor capacitance effect is included and the resonant behavior can be easily determined. After stablished, the FSS equivalent circuit can be applied even for a different varactor. In order to validate the proposed modeling, the equivalent circuit of a reconfigurable FSS using the varactor SMV1231 ( 0.466 pF $\left.\leq C_{v} \leq 2.35 \mathrm{pF}\right)$ is obtained. The equivalent circuit results are compared to numerical (ANSYS) and measured results, verifying a good agreement. Following, for the same geometry, the equivalent circuit is applied to a reconfigurable FSS using the SMV1234 varactor $(1.32 \mathrm{pF} \leq$ $C_{v} \leq 9.63 \mathrm{pF}$ ) and once more a good agreement between the results is observed, indicating the applicability of the proposed modeling, which is especially attractive for optimization process.
\end{abstract}

Index Terms - Four arms star, modeling, reconfigurable FSS, varactor.

\section{INTRODUCTION}

Frequency selective surfaces, FSS, consist of conductive patches or aperture elements, etched on a dielectric substrate, arranged in a planar periodic structure, providing filtering properties, which frequency response depends on the polarization of the incident wave, the geometry and periodicity of the elements within the FSS structure, and the substrate thickness and permittivity, Fig. 1. As FSS can pass or block electromagnetic waves in free space for different frequencies, they are also known as spatial filters [1]-[5]. FSS have been investigated for more than five decades, with applications from microwave to terahertz frequencies. Initially, FSS were employed in radomes, absorbers and dualband antennas sub-reflectors systems [1]-[4]. Nowadays, FSS have been reported in diverse applications, such as flat lens [6], [7], radar-cross-section-reduction [8], [9], polarization converters [10], [11], smart 
antennas [12], [13], and wireless security [14], [15].

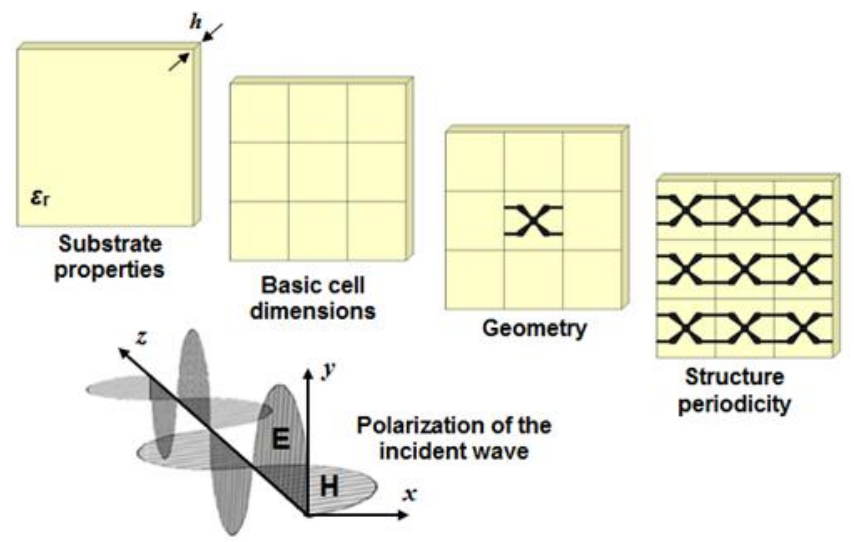

Fig. 1. FSS geometry and parameters that affect the FSS frequency response, [5].

When reconfigurable FSS are desired, two main approaches are considered: mechanical and electronic tuning. In the mechanical tuning authors exploit mechanical modifications, such as stretching, folding or rotate the basic element to achieve the frequency tuning [3],[16]-[18]. In the electronic tuning, discrete components, such as varactors, PIN diodes or MEMS switches, are incorporated in the FSS basic geometry [3], [5], [19]-[21]. Reconfigurable FSS have been especially attractive for implementation of smart antennas, an increasing demand of the wireless communication systems [3], [12], [13], [22], [23]. The electronic reconfigurability can be discrete or continuously. In discrete manner, the FSS assumes a limited number of frequency responses, and PIN diodes or MEMS switches are commonly used. Usually, a FSS with continuously variable frequency response is obtained by the use of varactors as tuning components.

Varactors are PN junction diodes in which the depletion region that is formed at the junction acts as a nearly-ideal insulator, which separates the highly-doped anode from the cathode layer, thus forming a parallel plate capacitor, which capacitance can be controlled by the reverse bias voltage [24]. Despite a more accurate model of the varactor includes inductances and resistances, Fig. 2, in many applications a simplified model, considering only the variable capacitance, can be adopted. Reconfigurable FSS frequency response, including varactor effects and bias lines, can be numerically simulated using commercial software [13], [25], [26]. However, the required computational processing time imposes limits, principally for optimization techniques. In this paper we introduced a modeling procedure from which the FSS equivalent circuit is obtained. Then, the varactor capacitance effect is included and the continuously reconfigurable FSS can be easily characterized. To the best of the authors' knowledge, this is the first time that the equivalent circuit of the FSS based on four arms star geometry is described. Furthermore, the proposed modeling procedure may be applied to other FSS geometries.

After introducing the FSS and varactors basics in this Section, four arms star geometry and FSS 
equivalent circuit are described in Section II. The proposed modeling, including numerical and measured results, is detailed in Section III. The procedures, results and conclusions are summarized in Section IV.

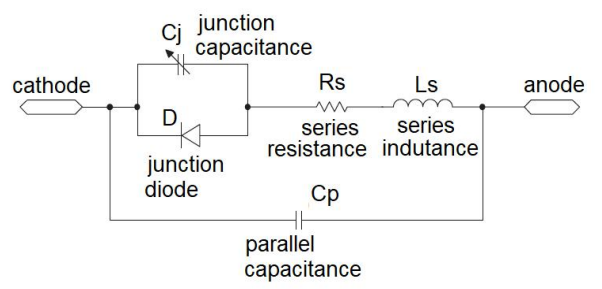

a) Detailed model

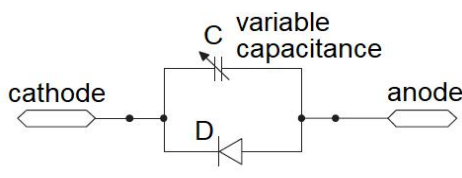

b) Variable capacitance model

Fig. 2. Varactor models.

\section{FOUR ARMS STAR GEOMETRY AND FSS EQUIVALENT CIRCUIT}

Four arms star geometry was introduced in [27], with very interesting characteristics, such as miniaturization and switching. In Fig. 3, the four arms star geometry is depicted and the procedure to obtain it is detailed in [5], [19], [21]. Without the gap, Fig. 3(a), it is polarization independent. With the gap insertion, Fig. 3(b) and Fig. 3(c), the geometry becomes polarization dependent and only the frequency response for the $y$ polarization is affected by the presence of the varactor. Including the bias lines, Fig. 3(d), basic cell is complete. If the insertion of the varactor is not considered, the FSS has a band-stop frequency response, as it is shown in Fig. 4.

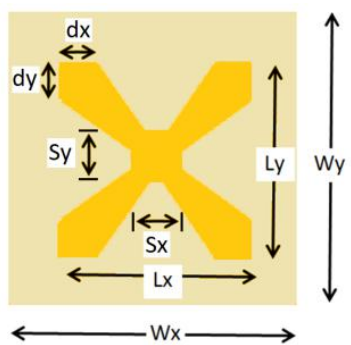

a) Geometry dimensions, without gap

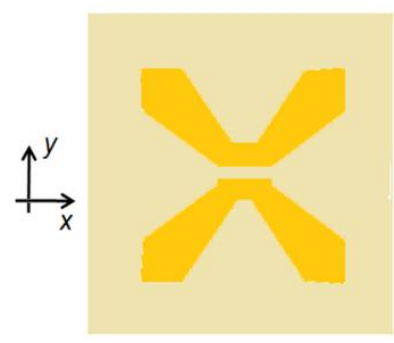

c) Geometry with gap

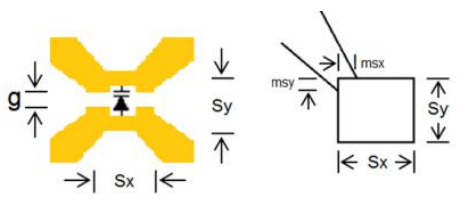

b) Gap details

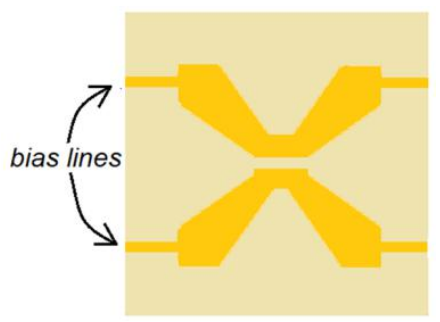

d) Geometry with gap and bias lines

Fig. 3. Four arms star geometry.

Without the gap, Fig. 3(a), the FSS resonant frequency can be approximately determined by (1), with good results, principally for $h \ll \lambda_{0}$, [5], [19], [21]. 


$$
f_{\text {res }}(G H z)=\frac{0.3}{2 L_{e f e}}
$$

in which $L_{e f e}=L x+L y$.

With the gap and the bias lines, Fig. 3 (d), roughly speaking, the resonant frequency, $f_{\text {res-gb }}$, can be estimated by (2).

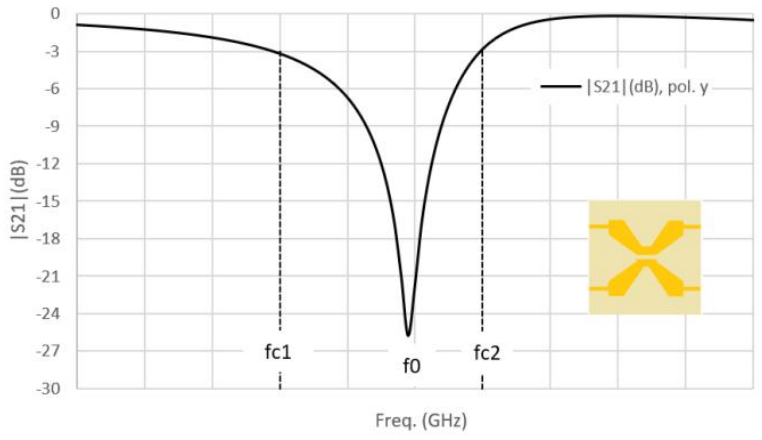

a) Typical frequency response - y polarization

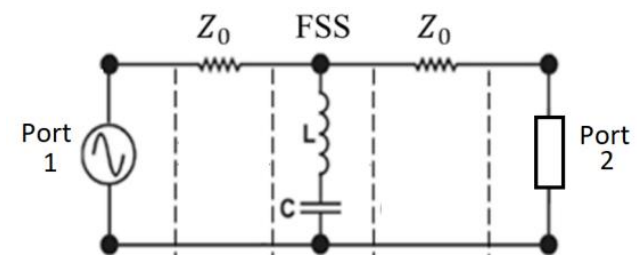

b) Equivalent circuit

Fig. 4. Typical four arms star FSS frequency response, y polarization.

$$
1.5 f_{\text {res }} \leq f_{\text {res }-g b}(G H z) \leq 2.0 f_{\text {res }},
$$

Despite the resonant frequencies obtained by (1) and (2) are not exact, these equations are specially interesting as a first step for a numerical optimization.

Taking into account the FSS frequency response and its equivalent circuit, Fig. 4, the following equations can be obtained.

$$
\begin{gathered}
f_{\text {res }-g b}=f_{0}=\frac{1}{2 \pi \sqrt{L C}} \\
C=\frac{\left|1-\left(\frac{f_{C}}{f_{0}}\right)^{2}\right|}{2 \pi f_{C} Z_{0}}
\end{gathered}
$$

Considering $Z_{0}$ known, $L$ and $C$ can be effortlessly determined. Inserting the varactor, a series variable capacitance is introduced, Fig. 5 , in which the equivalent capacitance is given by (5).

$$
C_{e q}=\frac{C \times C_{v}}{C+C_{v}}
$$

Note that if $C_{v} \gg C, C_{e q} \rightarrow C$. Otherwise, if $C_{v} \ll C, C_{e q} \rightarrow C_{v}$. Therefore, in order to obtain a variation in the FSS frequency response, the varactor must present a capacitance small enough when compared to the FSS intrinsic capacitance, as it will be verified numerically and experimentally in the next Section. 

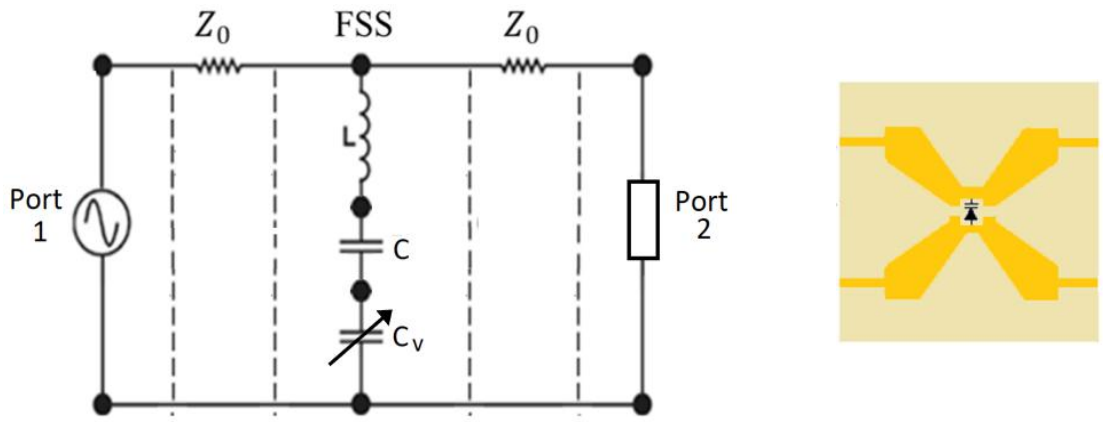

Fig. 5. FSS equivalent circuit considering the varactor insertion.

\section{MODELING THE FSS RESONANT BEHAVIOR}

As previously mentioned, in the proposed modeling it is presumed available a set of measured or numerical data, with frequency responses for different varactor capacitance values. Thus, initially it is described how numerical and measured results were acquired.

Numerical results were obtained using the commercial software ANSYS. The measured results were obtained at the GTEMA/IFPB microwave measurements laboratory using an Agilent VNA E5071C two ports vector network analyzer, two A.H. Systems double ridge horn antennas SAS-571, a Keysight E3633A power supply, and a measurement window as shown in Fig. 6. The wave incidence is considered normal to the reconfigurable FSS.

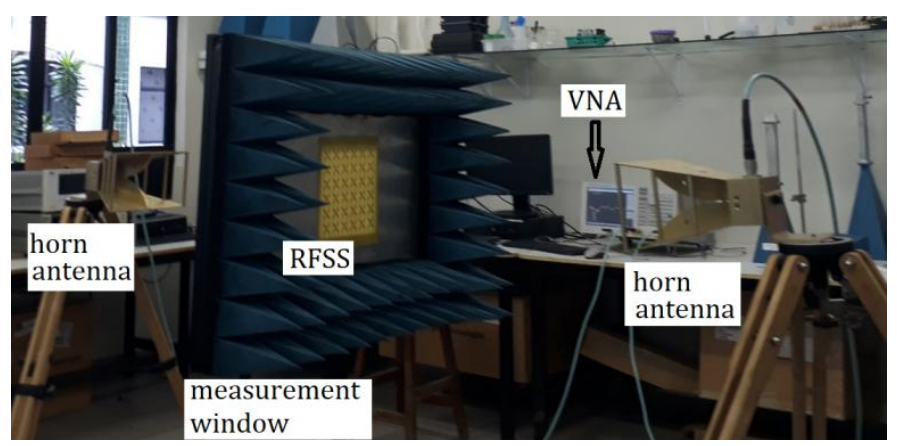

Fig. 6. Measurement setup.

The reconfigurable FSS based on four arms star geometry was designed and fabricated using a low cost fiber-glass substrate FR-4 ( $\varepsilon_{r}=4.4$, loss tangent 0.02 , thickness $1.2 \mathrm{~mm}, 35 \mu \mathrm{m}$ copper thickness) with the following basic cell dimensions: $W_{x}=W_{y}=30 \mathrm{~mm}, L_{x}=L_{y}=20 \mathrm{~mm}$, $S_{x}=S_{y}=d_{x}=d_{y}=3 \mathrm{~mm}, m_{x}=m_{y}=g=1 \mathrm{~mm}$, as depicted in Fig. 3. The whole reconfigurable FSS has $6 \times 6$ basic cells, arranged in 6 lines, with $180 \mathrm{~mm} \times 180 \mathrm{~mm}$. Despite the basic cell lines could be individually controlled, in this work they were connected. The resistor R, 150 $\Omega$, was introduced as a protection to avoid any damage due to a wrong connection. The voltage applied in varactors is directly controlled by the power supply. A photograph of the fabricated FSS is presented in Fig. 7. The used varactor is SMV1231-079LF [28], which Capacitance $(\mathrm{pF}) \times$ Reverse voltage (V) curve is presented in Fig. 8. 


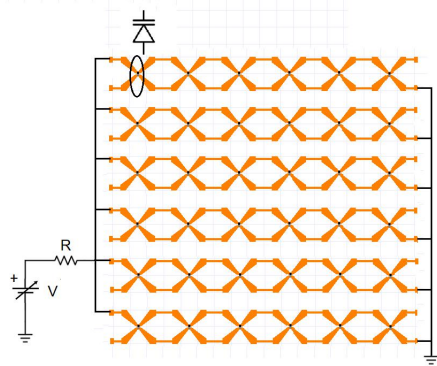

a) Reconfigurable FSS drawing

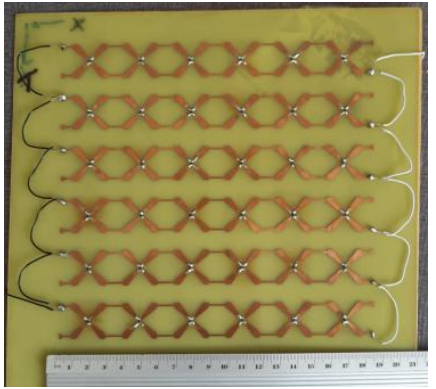

b) Fabricated prototype

Fig. 7. Continuously reconfigurable FSS.

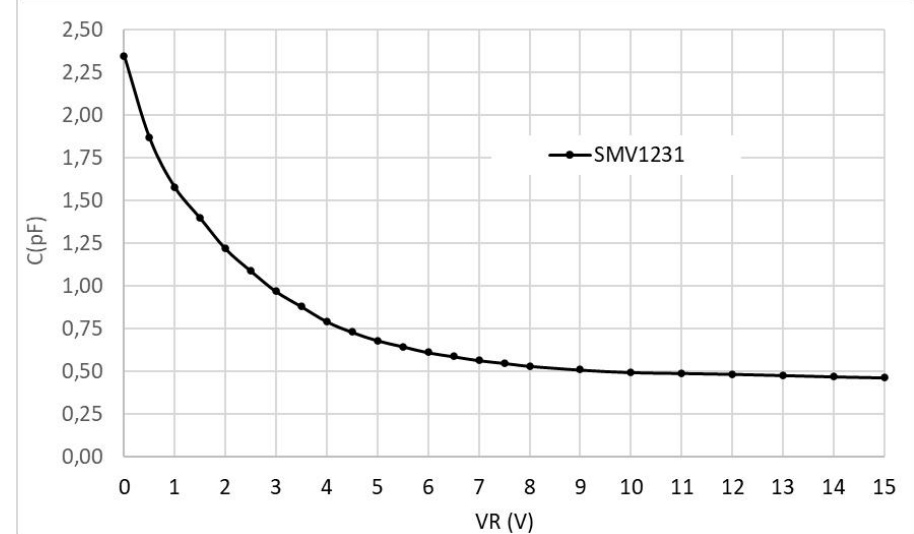

Fig. 8. Varactor SMV1231-079LF, Capacitance $(\mathrm{pF}) \times$ Reverse voltage $(V)$ curve [28]

Numerical results for the FSS frequency response, without the varactor insertion, are presented in Fig. 9-Fig. 11. In Fig. 9, only the four arms star geometry is considered, with $x$ and $y$ polarization showing the same frequency response. The resonant frequency obtained by (1) is $3.75 \mathrm{GHz}$, a difference of $7.4 \%$ when compared to the numerical result, $4.05 \mathrm{GHz}$, a good approximation for a numerical optimization first step. Fig. 10 presents the FSS frequency response for the four arms star geometry with the gap. As expected, the $x$ polarization frequency response remains practically unchanged. The frequency response, after adding the bias lines, is shown in Fig. 11, with a resonant frequency of $6.33 \mathrm{GHz}$ for $y$ polarization. In this case, resonant frequency for $x$ polarization is out of the frequency range of interest.
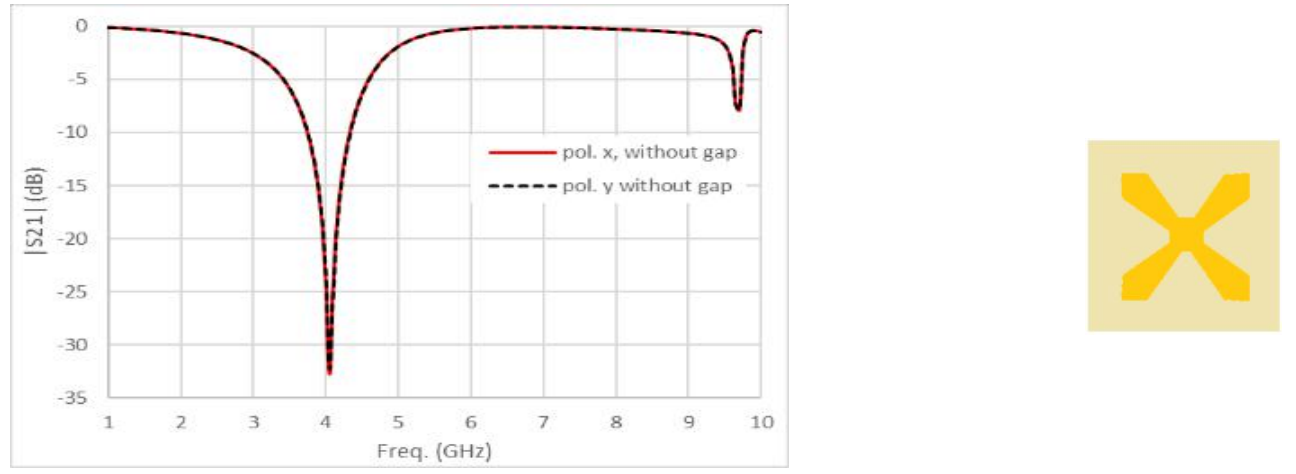

Fig. 9. FSS frequency response - Four arms star geometry. 


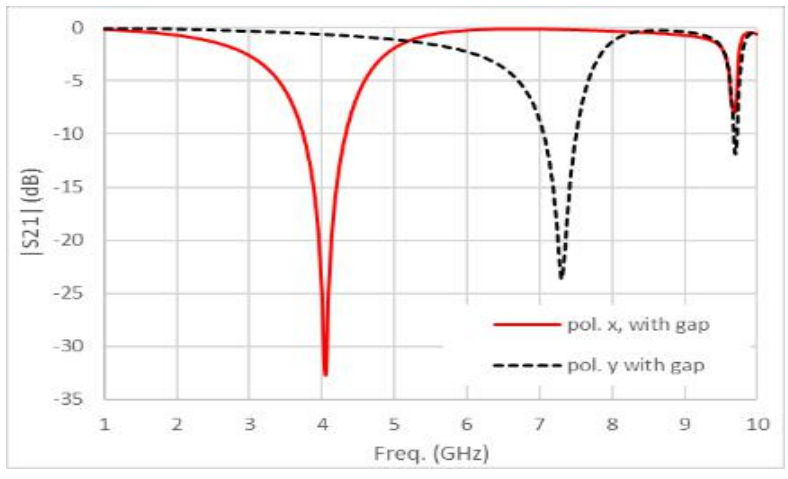

Fig. 10. FSS frequency response - Four arms star geometry with gap.

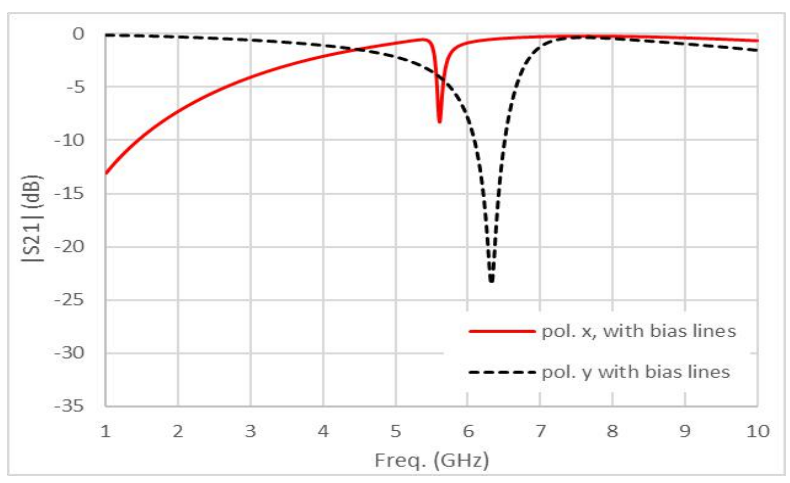

Fig. 11. FSS frequency response - Four arms star geometry with bias lines.

In Fig. 12, for $y$ polarization, numerical and measured results for the frequency response are presented for different reverse voltages, $1 \mathrm{~V}(1.58 \mathrm{pF}), 5 \mathrm{~V}(0.68 \mathrm{pF})$, and $10 \mathrm{~V}(0.50 \mathrm{pF})$, considering the varactor SMV1231. Each reverse voltage is associated to a capacitance in accordance with Fig. 8 . This capacitance value was used in the lumped RLC boundary (ANSYS) to numerically determine the FSS frequency response. For the reverse voltages, $1 \mathrm{~V}, 5 \mathrm{~V}$ and $10 \mathrm{~V}$, the measured/numerical resonant frequencies are, respectively, $3.89 \mathrm{GHz} / 4.01 \mathrm{GHz}, 4.31 \mathrm{GHz} / 4.48 \mathrm{GHz}, 4.79 \mathrm{GHz} / 4.70 \mathrm{GHz}$, which correspond to a maximum difference of $3.8 \%$, a good agreement, despite the resonance intensity differences.

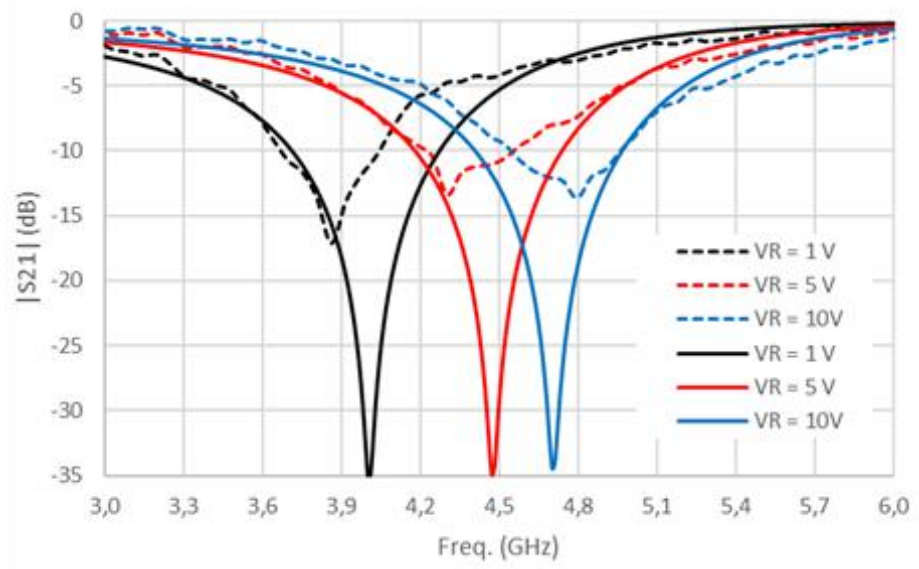

Fig. 12. $|\mathrm{S} 21|(\mathrm{dB}) \times$ Frequency $(\mathrm{GHz})$, for different reverse voltages, varactor SMV1231, numerical results (continuous lines), measured results (dashed lines). 
Journal of Microwaves, Optoelectronics and Electromagnetic Applications, Vol. 19, No. 3, September 2020 DOI: http://dx.doi.org/10.1590/2179-10742020v19i3930

In Fig. 13 the reconfigurable FSS resonant frequency is presented as a function of the reverse voltage, for the varactor SMV1231. This result is superimposed with the capacitance curve. A first feature to be highlighted is the good agreement between numerical and measured results, both curves presenting a similar behavior. Furthermore, a continuous variation of the resonant frequency from $3.750 \mathrm{GHz}$ to $4.795 \mathrm{GHz}$ is achieved (measured), a bandwidth of $1.045 \mathrm{GHz}$, which is very interesting. When the capacitance achieves the minimum value saturation, around $10 \mathrm{~V}(0.50 \mathrm{pF})$, it can be observed a similar behavior of the resonant frequency, limiting the reconfigurable FSS resonance to 4.795 GHz, measured/4.700 GHz, numerical. Fig. 14 presents the same result, but now with the resonant frequency as a function of the capacitance.

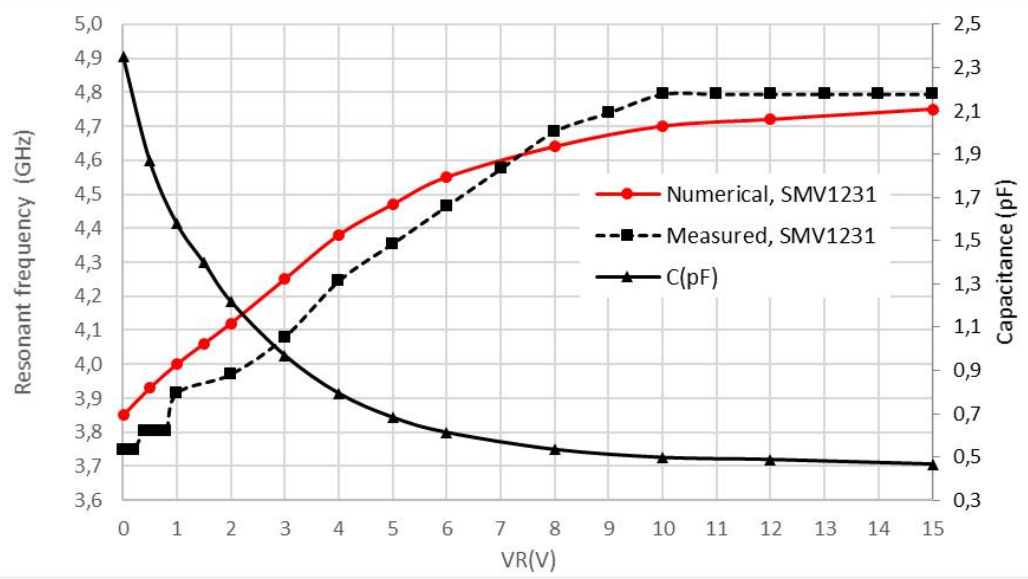

Fig. 13. Resonant frequency $(\mathrm{GHz}) \times$ Reverse voltage $(\mathrm{V})$, varactor SMV1231.

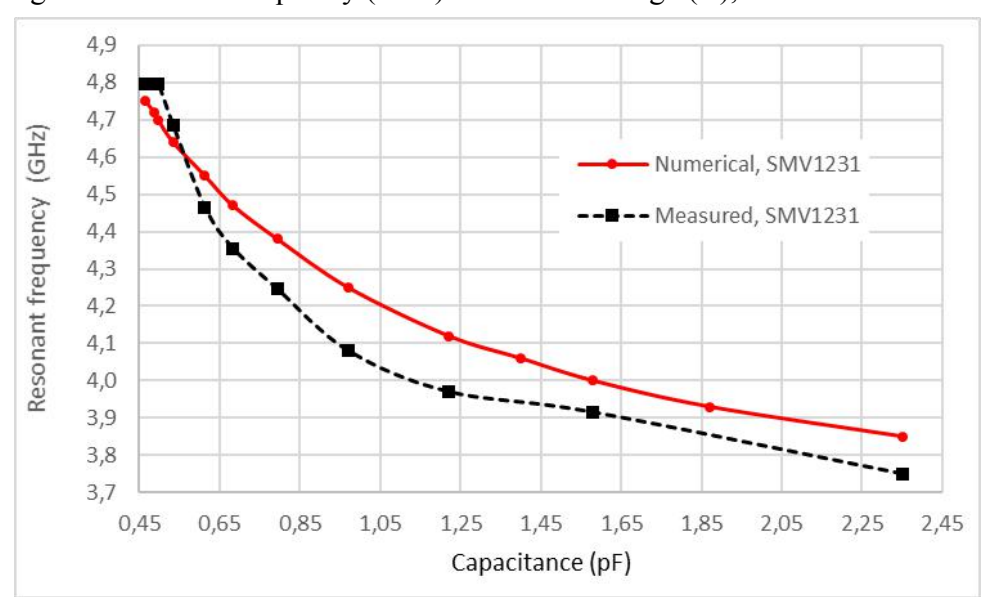

Fig. 14. Resonant frequency $(\mathrm{GHz}) \times$ Capacitance $(\mathrm{pF})$, varactor SMV1231.

In order to achieve the FSS equivalent circuit, Fig. 5, it is necessary to determine the adequate values of $Z_{0}, \mathrm{C}$ and $\mathrm{L}$. Considering available a set of measured or numerical data, with frequency responses for different varactor capacitance values, the following steps are adopted:

1-The cutoff frequency, $\mathrm{f}_{\mathrm{C}}$, and the resonant frequency, $\mathrm{f}_{0}$, are extracted for the lowest varactor capacitance, $\mathrm{C}_{\mathrm{v}}$ (highest reverse voltage).

2-An impedance value $Z_{0}$ is assumed and from (3) and (4), $C_{e q}$ and $L$ are calculated. Note that this capacitance includes the varactor capacitance and the FSS intrinsic capacitance. 
Journal of Microwaves, Optoelectronics and Electromagnetic Applications, Vol. 19, No. 3, September 2020 DOI: http://dx.doi.org/10.1590/2179-10742020v19i3930

3-From (5) the FSS capacitance, C, is determined.

4-With the FSS equivalent circuit, from (3), the resonant frequencies are calculated for the other varactor capacitance values.

5-If the obtained resonant frequency curve fits the available measured/numerical curve, the procedure is completed.

6-If a good fitting is not achieved, a new $Z_{0}$ is assumed and the procedure is repeated, step 2.

It must be highlighted that after the FSS capacitance and inductance be obtained, a new varactor can be inserted and the reconfigurable FSS frequency response can be effortless calculated. To exemplify the proposed procedure, be considered the measured frequency response for $\mathrm{VR}=10.0 \mathrm{~V}$, $C_{v}=0.497 \mathrm{pF}$, in which $f_{C}=3.86 \mathrm{GHz}$ and $f_{0}=4.795 \mathrm{GHz}$. Resonant frequency curves are shown in Fig. 15 for different $\mathrm{Z}_{0}$ values. Choosing $\mathrm{Z}_{0}=62.5 \Omega$, we obtain $C_{e q}=0.231 \mathrm{pF}$, $L=4.77 \mathrm{nH}, C=0.430 \mathrm{pF}$. Fig. 16 presents the frequency responses for different reverse voltages, as shown Fig. 12, but now including the frequency responses obtained with the FSS equivalent circuit. When compared to numerical and measured results, equivalent circuit results present a good agreement.

Still considering the equivalent circuit obtained, and the resonant frequency curve, Fig. 15, another interesting result is the interpolation equation for the resonant frequency as a function of $C_{v}$ :

$$
f_{\text {res }}\left(C_{v}\right)(G H z)=0.156 C_{v}{ }^{4}-1.153 C_{v}{ }^{3}+3.232 C_{v}{ }^{2}-4.289 C_{v}+
$$

If $C_{v}=0 \mathrm{pF}$, we get the resonant frequency of the FSS without the varactor insertion, in this case $6.240 \mathrm{GHz}$, a very good result when compared to the numerical one, $6.33 \mathrm{GHz}$, as shown in Fig. 11 .

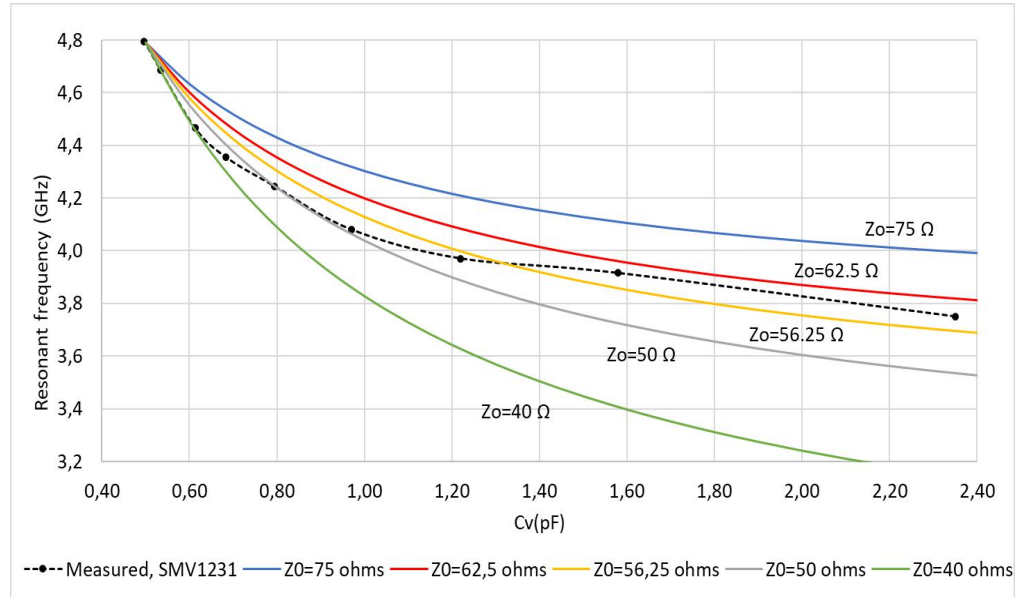

Fig. 15. Resonant frequency $(\mathrm{GHz}) \times$ Capacitance $(\mathrm{pF})$, varactor SMV1231, for different $\mathrm{Z}_{0}$ values. 

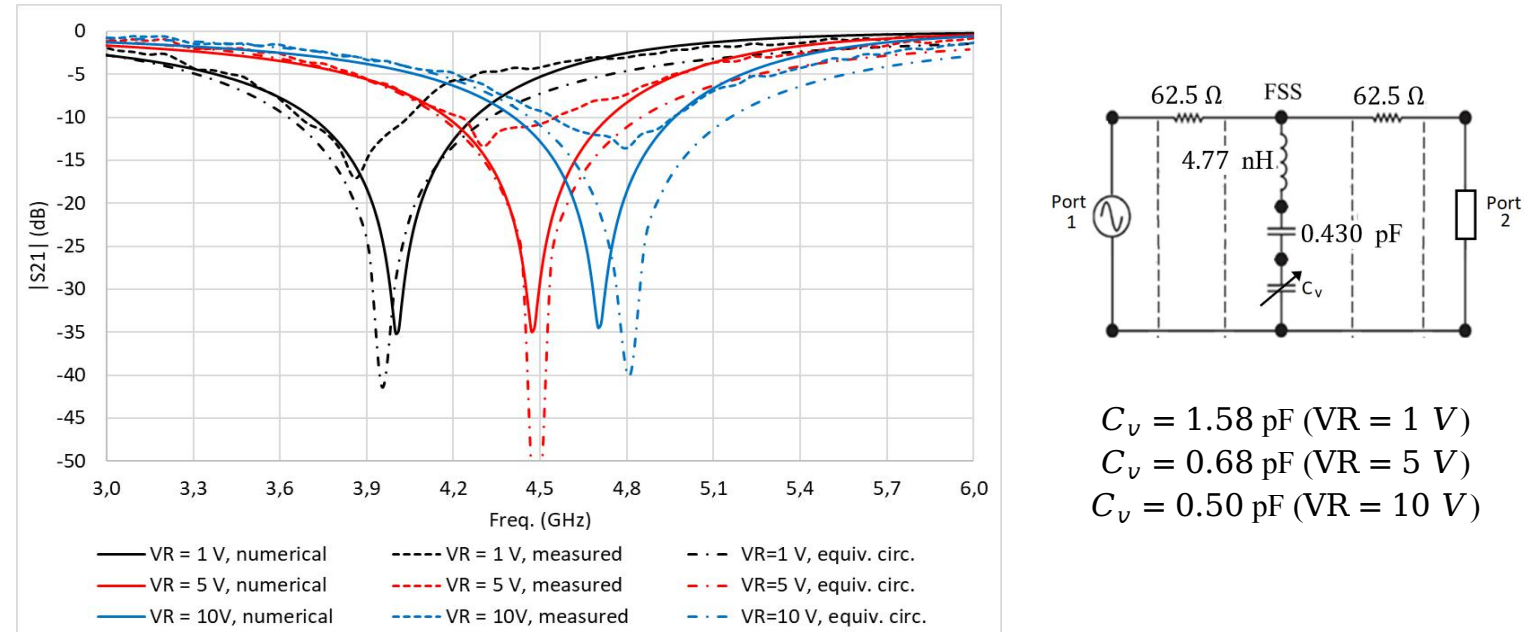

$$
\begin{gathered}
C_{v}=1.58 \mathrm{pF}(\mathrm{VR}=1 \mathrm{~V}) \\
C_{v}=0.68 \mathrm{pF}(\mathrm{VR}=5 \mathrm{~V}) \\
C_{v}=0.50 \mathrm{pF}(\mathrm{VR}=10 \mathrm{~V})
\end{gathered}
$$

Fig. 16. $|\mathrm{S} 21|(\mathrm{dB}) \times$ Frequency $(\mathrm{GHz})$, for different reverse voltages, varactor SMV1231, numerical results (continuous lines), measured results (dashed lines), equivalent circuit (dashed dotted lines).

In order to evaluate the equivalent circuit for another varactor, be considered the results present in [21], in which the same FSS geometry is adopted, but using a varactor SMV1234 [28], that presents the resonant frequency curves as shown in Fig. 17. As the FSS equivalent circuit has been determined, the frequency response can be effortless determined, Fig. 18. Fig. 19 presents the frequency responses for different reverse voltages, including the frequency responses obtained with the FSS equivalent circuit. When compared to numerical and measured results, equivalent circuit results present a good agreement.

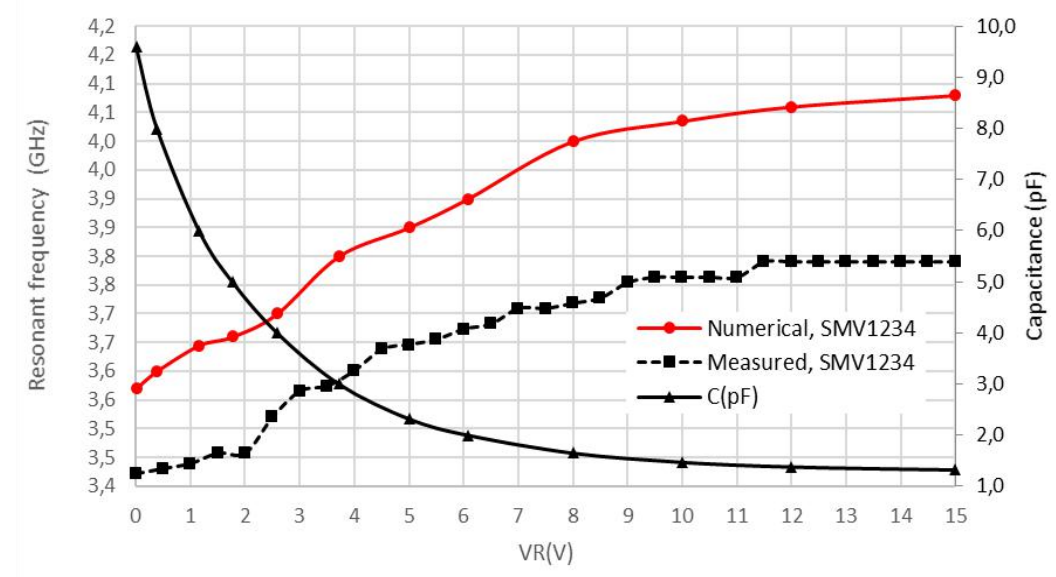

Fig. 17. Resonant frequency $(\mathrm{GHz}) \times$ Reverse voltage $(\mathrm{V})$, varactor SMV1234, [21] 


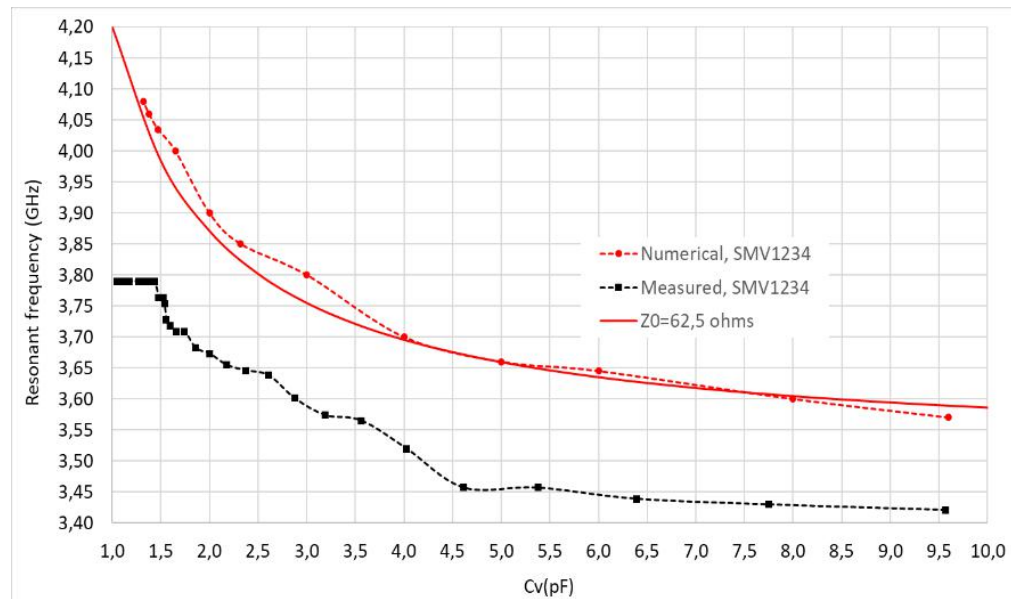

Fig. 18. Resonant frequency $(\mathrm{GHz}) \times$ Capacitance $(\mathrm{pF})$, varactor SMV1234.
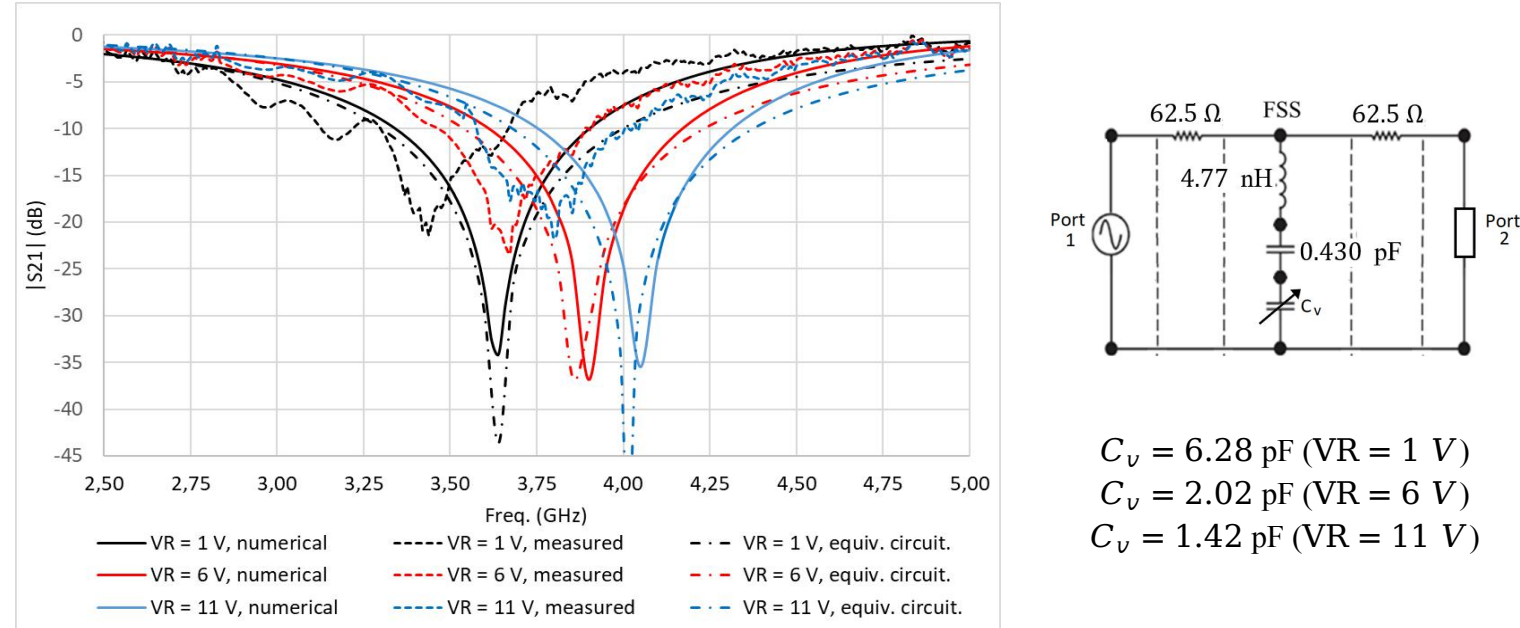

$C_{v}=6.28 \mathrm{pF}(\mathrm{VR}=1 \mathrm{~V})$

$C_{v}=2.02 \mathrm{pF}(\mathrm{VR}=6 \mathrm{~V})$

$C_{v}=1.42 \mathrm{pF}(\mathrm{VR}=11 \mathrm{~V})$

Fig. 19. $|\mathrm{S} 21|(\mathrm{dB}) \times$ Frequency $(\mathrm{GHz})$, for different reverse voltages, varactor SMV1234, numerical results (continuous lines), measured results (dashed lines), equivalent circuit (dashed dotted lines).

\section{CONCLUSiOnS}

A modeling of the resonant behavior of a continuously reconfigurable FSS based on the four arms star geometry was presented in this paper. Initially the FSS and the four arms geometry principles were described. The FSS reconfigurability is achieved electronically, by the use of varactors, for which the adopted model was also described. The proposed modeling take account the availability of initial set of FSS frequency response for different varactor capacitances, which can be obtained numerically or experimentally. However, after the FSS modeling has been carried out, the obtained equivalent circuit can be applied to different capacitance values, and new frequency responses can be determined in fast manner.The modelling procedure was described and successfully applied to a reconfigurable FSS using the SMV1231 varactor $\left(0.466 \mathrm{pF} \leq C_{v} \leq 2.35 \mathrm{pF}\right)$. The equivalent circuits results were compared to numerical (ANSYS) and measured results, verifying a good agreement. After, for the same geometry, the equivalent circuit was applied to a reconfigurable FSS using the SMV1234 varactor (1.32 pF $\left.\leq C_{v} \leq 9.63 \mathrm{pF}\right)$. Once again, a good agreement was observed between 
Journal of Microwaves, Optoelectronics and Electromagnetic Applications, Vol. 19, No. 3, September 2020 DOI: http://dx.doi.org/10.1590/2179-10742020v19i3930

numerical, measured and equivalent circuit results, indicating the applicability of the proposed modeling.

The proposed modeling is especially attractive for optimization techniques, since after the FSS equivalent circuit has been achieved, the varactor capacitance can be varied and different frequency responses can be readily obtained.

\section{ACKNOWLEDGMENT}

The authors would like to thank to Leanne Vincent, from Skyworks, by the provided varactors. This work was supported in part by the CNPq (Project 407028/2016-1) and CAPES, Brazilian Federal Agencies, and by the IFPB, Federal Institute of Paraíba (PPGEE, PRPIPG 01/2019).

\section{REFERENCES}

[1] B. A. Munk, Frequency Selective Surfaces - Theory and Design, New York: Wiley, 2000.

[2] John C. Vardaxoglou, Frequency Selective Surfaces - Analysis and Design, Research Studies Press, England, 1997.

[3] Ravi Panwar, Jung Ryul Lee, "Progress in frequency selective surface-based smart electromagnetic structures: A critical review," Aerospace Science and Technology, vol. 66, pp. 216-234, 2017.

[4] Rana Sadaf Anwar, Lingfeng Mao and Huansheng Ning, "Frequency Selective Surfaces: A Review," Applied Sciences, vol. 8 , no. 9, pp.1689, Sep. 2018

[5] Amanda Gomes Barboza, Desenvolvimento de Superfície Seletiva em Frequência Reconfigurável Utilizando Varactores [Development of Reconfigurable Frequency Selective Surface Using Varactors], in Portuguese, M.Sc. thesis, Postgraduate Program in Electrical Engineering, Federal Institute of Paraíba, IFPB, João Pessoa, Paraíba, 2020.

[6] S. Pandey, R. K. Arya and R. Mittra, "Flat lens design using space-qualifiable multilayer frequency selective surfaces," 2016 IEEE International Symposium on Antennas and Propagation (APSURSI), Fajardo, pp. 1643-1644, 2016. doi: 10.1109/APS.2016.7696528.

[7] Y. Suo, H. Wang and W. Li, "C-Band Multi-Beam Planar Lens Antenna Based on Frequency Selective Surface," 2019 IEEE International Symposium on Antennas and Propagation and USNC-URSI Radio Science Meeting, Atlanta, GA, USA, pp. 727-728, 2019.

[8] V. K. Chakradhary, H. Bhusan Baskey and M. J. Akhtar, "Novel X-band Microwave Absorber Based on Carbon Nanofiber Integrated with the FSS Structure for RCS Reduction of Strategic Targets," 2018 IEEE MTT-S International Microwave and RF Conference (IMaRC), Kolkata, India, pp. 1-4, 2018.

[9] M. Pazokian, N. Komjani and M. Karimipour, "Broadband RCS Reduction of Microstrip Antenna Using Coding Frequency Selective Surface," in IEEE Antennas and Wireless Propagation Letters, vol. 17, no. 8, pp. 1382-1385, Aug. 2018.

[10] C. Yang, P. Liu and X. Zhu, "Circularly Polarized Microstrip Patch Antenna Array Based on FSS Polarization Converter," 2019 International Symposium on Antennas and Propagation (ISAP), Xi'an, China, pp. 1-3, 2019.

[11] F. Costa, S. Genovesi, A. Monorchio, S. Zhang, Y. Cui and J. Liu, "Broadband Linear to Circular Reflection Polarization Converter," 2019 IEEE International Symposium on Antennas and Propagation and USNC-URSI Radio Science Meeting, Atlanta, GA, USA, pp. 1823-1824, 2019.

[12] J. Li, Q. Zeng, R. Liu and T. A. Denidni, "A Gain Enhancement and Flexible Control of Beam Numbers Antenna Based on Frequency Selective Surfaces," in IEEE Access, vol. 6, pp. 6082-6091, 2018.

[13] Chao Gu, Steven Gao, Benito Sanz-Izquierdo, Edward A. Parker, Wenting Li, Xuexia Yang, and Zhiqun Cheng, "Frequency-Agile Beam-Switchable Antenna," in IEEE Transactions on Antennas and Propagation, vol. 65, no. 8, pp. 3819-3826, Aug. 2017.

[14] S. Yadav, C. P. Jain and M. M. Sharma, "Smartphone Frequency Shielding With Penta-Bandstop FSS for Security and Electromagnetic Health Applications," in IEEE Transactions on Electromagnetic Compatibility, vol. 61, no. 3, pp. 887892, June 2019.

[15] X. Xiong, Weijun Hong, Zhentao Zhao, Li Deng and Shufang Li, "WiFi band-stop FSS for increased privacy protection in smart building," 2015 IEEE 6th International Symposium on Microwave, Antenna, Propagation, and EMC Technologies (MAPE), Shanghai, pp. 826-828, 2015.

[16] S. N. Azemi, K. Ghorbani and W. S. T. Rowe, "A Reconfigurable FSS Using a Spring Resonator Element," in IEEE Antennas and Wireless Propagation Letters, vol. 12, pp. 781-784, 2013.

[17] D. Ferreira, I. Cuiñas, R. F. S. Caldeirinha and T. R. Fernandes, "3-D Mechanically Tunable Square Slot FSS," in IEEE Transactions on Antennas and Propagation, vol. 65, no. 1, pp. 242-250, Jan. 2017. 
Journal of Microwaves, Optoelectronics and Electromagnetic Applications, Vol. 19, No. 3, September 2020

DOI: http://dx.doi.org/10.1590/2179-10742020v19i3930

[18] S. A. B, E. F. Sundarsingh and V. S. Ramalingam, "Mechanically Reconfigurable Frequency Selective Surface for RF Shielding in Indoor Wireless Environment," in IEEE Transactions on Electromagnetic Compatibility. doi: 10.1109/TEMC.2020.2983899.

[19] D. F. Mamedes, A. Gomes Neto, J. C. e Silva and J. Bornemann, "Design of reconfigurable frequency-selective surfaces including the PIN diode threshold region," in IET Microwaves, Antennas \& Propagation, vol. 12, no. 9, pp. 1483-1486, 2018.

[20] Z. Lu, J. She and X. Yan, "A dual-band reconfigurable FSS composite structure based on MEMS switches," 2016 11th International Symposium on Antennas, Propagation and EM Theory (ISAPE), Guilin, pp. 630-632, 2016.

[21] A. G. Neto, J. C. e Silva, A. G. Barboza, D. F. Mamedes, I. B. G. Coutinho and M. de Oliveira Alencar, "VaractorTunable Four Arms Star Bandstop FSS With a Very Simple Bias Circuit," 2019 13th European Conference on Antennas and Propagation (EuCAP), Krakow, Poland, pp. 1-5, 2019.

[22] M. A. Erni, G. G. Mendoza and S. M. Cheng, "Design and Implementation of a 3D Beam Steering Antenna for Cellular Frequencies," 2019 IEEE Asia-Pacific Microwave Conference (APMC), Singapore, Singapore, pp. 1524-1526, 2019.

[23] G. H. Elzwawi, A. Kesavan, R. Alwahishi and T. A. Denidni, "A New Corner-Reflector Antenna With Tunable Gain Based on Active Frequency Selective Surfaces," in IEEE Open Journal of Antennas and Propagation, vol. 1, pp. 88-94, 2020.

[24] Skyworks, Varactors Diodes - Application Notes, Available: https://www.skyworksinc.com//media/SkyWorks/Documents/Products /1-100/200824A.pdf

[25] Lin, B. Q., Guo, J., Chu, P., Li, N., Wu, N., Liu, X. "Varactor-tunable frequency selective surface with an appropriate embedded bias network," Radio Science, vol. 53, pp. 535-543. 2018.

[26] Lin Bao-Qin, Qu Shao-Bo, Tong Chuang-Ming, Zhou Hang, Zhang Heng-Yang, and Li Wei "Varactor-tunable frequency selective surface with an embedded bias network," Chinese Phys. B, vol. 22, No. 9, pp. 094103-1-094103-4, 2013.

[27] A. G. Neto, J. N. de Carvalho, A. N. da Silva, H. de Paiva Almeida Ferreira, I. S. S. Lima and J. I. Fernandes, "Four arms star: An useful geometry for switchable FSS," 2013 SBMO/IEEE MTT-S International Microwave \& Optoelectronics Conference (IMOC), Rio de Janeiro, pp. 1-5, Aug. 2013.

[28] Skyworks Solutions, Inc., SMV123x Series: Hyperabrupt Junction Tuning Varactors - Data Sheet, Note, July 28, 2016

[29] https://www.multisim.com/ 